\title{
Colorimetric study of traditional varnishes applied on paper exposing to aging tests
}

\section{Estudio colorimétrico de barnices tradicionales aplicados sobre papel sometidos a ensayos de envejecimiento acelerado}

\author{
M. Durbán ${ }^{1}$, T. Espejo*1, A. López-Montes ${ }^{1}$, M.R. Blanc ${ }^{2}$ \\ 1. Departamento de Pintura, Universidad de Granada \\ 2. Departamento de Química Analítica, Universidad de Granada \\ ${ }^{(*)}$ E-mail: tespejo@ugr.es
}

Received: 18/12/2018 Accepted: 04/03/2019

DOI: $10.7149 /$ OPA.52.1.51018

\begin{abstract}
:
The use of varnishes as a final coat in graphic work fulfills an aesthetic and protective function. Over time, or due to adverse conditions, these varnishes undergo a series of degradations in their color that can distort the original appearance of the underlying work. Knowing the chromatic variations of these materials is essential for their selection as a conservation varnish.

This work collects the results obtained from the colorimetric study carried out on a series of samples of paper with varnish exposing to different physical-chemical tests (humidity, immersion in water, dry heat and accelerated aging) tests that recreate adverse conditions to which this type of woks may be exposed as a consequence of its natural aging, the action of certain degradation factors or the restorative action. For this purpose, Somerset $100 \%$ cotton paper has been selected, which has been applied different varnishes of traditional use in graphic works: shellac, gum arabic, egg white, rosin and dammar. The color of these samples has been studied following the CIEL*a*b* 1976 system, calculating the colorimetric differences from the comparison between the reference samples and the samples after the physical-chemical tests.
\end{abstract}

Key words: colorimetry, varnish, paper, aging

\section{RESUMEN:}

El empleo de barnices como acabado final en obra gráfica cumple una función estética y protectora. Con el tiempo, o debido a factores adversos, estos barnices pueden sufrir una serie de alteraciones en su color, distorsionando el aspecto original de la obra subyacente. Conocer las variaciones cromáticas de estos materiales resulta esencial de cara a su selección como barniz de conservación.

Este trabajo recoge los resultados obtenidos del estudio colorimétrico llevado a cabo sobre una serie de probetas de papel barnizadas sometidas a distintos ensayos físico-químicos (humedad ambiental, inmersión en agua, calor seco y envejecimiento acelerado en cámara climática) que buscan recrear condiciones adversas a las que pueden verse sometidos este tipo de documentos como consecuencia de su envejecimiento natural, la acción de determinados factores de degradación o la acción restauradora. El papel seleccionado como soporte ha sido Somerset $100 \%$ algodón al que se le han aplicado distintos barnices de uso tradicional en obra gráfica: goma laca, goma arábiga, clara de huevo, colofonia y dammar). El color de estas probetas se ha estudiado siguiendo el sistema CIEL*a*b* 1976, calculando las diferencias colorimétricas antes y después de los ensayos físico-químicos y de envejecimiento.

Palabras clave: colorimetría, barniz, papel, envejecimiento. 


\section{REFERENCES AND LINKS / REFERENCIAS Y ENLACES}

[1] P.J. Young. "The Tale of the red-winged blackbird: a case study of varnish removal from a watercolor painting", The Book and Paper Group Annual, 89-96 (1999)

[2] S. Kroustallis. "Binding media in medieval manuscripts illumination: a source research", Revista de História da Arte, 113-125 (2011).

[3] A. Serrano. Los globos celestes y terrestres. Introducción a la restauración de globos, M. Domingo, I. Muíña (Eds.), Investigación, conservación y restauración de materiales y objetos cartográficos. Secretaría General Técnica, Madrid, 2011, pp. 81-96

[4] D. van der Reyden. "The History, Technology and Care of Globes: Case Study on the Technology and Conservation Treatment of Two NineteenthCentury Time Globes", The Paper Conservator, 21-30 (1988).

[5] S. Holden. "The development of lithographic cartography and the conservation treatment of a large varnish map", The Book and Paper Group Annual, 75-82 (1984).

[6] D. van der Reyden, D. Williams. "The technology and conservation treatment of a nineteenth century English papier-mâché chair", American Institute for the Conservation, 125-142 (1986)

[7] A. Treacy. "Reforming varnish on varnished Works of art on paper", AICC; Symposium, 117-136 (2006).

[8] J. Colbourne, B. Singer. "The removal of natural resin varnishes from hand-coloured oil printed media. In: Research in book and paper conservation in Europe: a state of the art. Verlag Berger Horn, Viena (2009).

[9] E. Mosier, D. van der Reyden, M. Baker. "The technology and treatment of an embosed, chromolithographic mechanical victorian Valentine card", The Book and Paper Group Annual, 10-25 (1992).

[10] C.E. Rogge. “The varnished truth: the récipes and reality of tintype coatings”, Journal of Cultural Heritage 15, 57-63 (2014).

[11] V. Horie. Materials for conservation. Organic consolidantes, adhesives and coatings. Nueva York, Routledge (2010).

[12] M. Matteini, A. Moles. La química en la restauración. San Sebastián, Nerea (2001).

[13] J. Mills, R. White. The organic chemistry of museum objects. Londres, Butterworth (1987).

[14] D. Ciofini, J. Striova, M. Camaiti, S. Siano. "Photo-oxidative kinetics of solvent and oil-based terpenoid varnishes", Polymer Degradation and Stability 123, 47-61 (2016).

[15] A. Villarquide. La pintura sobre tela I. San Sebastián, Nerea (2004).

[16] M. Castillo-Valdivia, A. López-Montes, T. Espejo, J. Vilchez, R. Blanc, R. (2001). “Caracterización de la goma arábiga y estudio de los cambios inducidos por envejecimiento artificial”, Conferencia Trienal del ICOM-CC, Lisboa, Portugal, 1-10 (2001).

[17] M. Huertas Torrejón. Materiales, procedimientos y técnicas pictóricas I. Madrid, Akal (2010).

[18] International Organization for Standarization (ISO). Pinturas y barnices. Determinación de la resistencia a la humedad. Parte 2: Método de exposición de probetas en atmósferas con condensación de agua. UNE-EN ISO 6270-2:2005.

[19] International Organization for Standarization (ISO). Pinturas y barnices. Determinación de la resistencia a líquidos. Parte 2: Método de inmersión en agua. UNE-EN ISO 2812-2:2007.

[20] International Organization for Standarization (ISO). Papel y cartón. Envejecimiento acelerado. Parte 1: Tratamiento con calor seco a $105^{\circ}$. UNE-EN ISO 57092-1: 2002.

[21] International Organization for Standarization (ISO). Pinturas y barnices. Método de exposición a fuentes luminosas de laboratorio. Parte 2: Lámparas de arco de xenon. UNE-EN ISO 16474-2:2013.

[22] International Organization for Standarization (ISO). Colorimetría. Parte 4: Espacio cromático L*a*b* CIE 1976. UNE-EN ISO 11664-4:2008.

[23] W. Mokrzycki, M, Tatol. Color difference Delta E- A survey. Machine Graphics and Vision (2011).

\section{Introducción}

Los barnices se han empleado habitualmente como capa final de protección en obra gráfica, ya sea sobre dibujos y pintura, grabados, fotografías o impresiones [1]. Las tipologías documentales y artísticas que responden a esta casuística son variadas. Los primeros ejemplos de obras barnizadas datan de la Edad Media, cuando las miniaturas podían ser barnizadas con los mismos materiales empleados como 
aglutinante de los pigmentos; este es el caso del barniz de clara de huevo [2]. A partir del siglo XVI, surge una tipología documental donde es habitual el uso de barnices. Es el caso de los globos terráqueos y celestes $[3,4]$, mapas y planos [5] a los que se aplicaban diferentes resinas para protegerlos de los daños derivados del uso y la manipulación. En el siglo XVIII, el interés por las lacas japonesas, conllevó la imitación de esa técnica en Europa sobre objetos realizados en madera o papel, empleando para ello barnices como la goma laca [6]. Sin embargo, la mayor parte de las obras gráficas barnizadas datan del siglo XIX, cuando los barnices se aplicaron no sólo con una función protectora, sino también estética, buscando imitar el acabado de una pintura al óleo en obras realizadas con otras técnicas como la litografía o la acuarela $[1,7,8]$. Surgen en este periodo nuevas tipologías gráficas en las que la presencia del barniz es habitual. Este es el caso de las cromolitografías — barnizadas con goma arábiga o goma laca, principalmente- [9], o determinados procesos fotográficos como el ferrotipo en los que es habitual la presencia de una película protectora a base de goma laca o de resina dammar [10].

Desde el punto de vista material, todos los barnices señalados son de naturaleza orgánica, pudiendo clasificarse en proteicos (clara de huevo), gomas naturales (goma arábiga) y resinas; estas últimas son las más abundantes, y se clasifican según su composición en resinas diterpénicas (colofonia), triterpénicas (dammar) y sesquiterpénicas (goma laca) [11,12,13].

Estos materiales pueden verse alterados debido a factores de degradación internos -envejecimiento natural - y externos -exposición a condiciones adversas o a tratamientos de conservación-, de modo que la función original de un barniz puede verse comprometida, poniendo en peligro la conservación de la obra o variando sus propiedades ópticas; así, el color puede alterarse, oscureciéndose y amarilleándose [14] modificando, en consecuencia, el aspecto original de la obra.

El objetivo de este estudio es determinar los efectos que distintos factores de alteración como la humedad, la temperatura y la radiación lumínica producen en el color de estos barnices, con el propósito de dotar al conservador-restaurador de una herramienta útil de trabajo que le permita tomar decisiones en relación con la utilización, eliminación o sustitución del barniz original tras el tratamiento. Para ello, se ha diseñado un estudio que ha partido de la preparación de una serie de probetas a las que se han aplicado cinco barnices, seleccionados por haber sido empleados históricamente en este tipo de obras - goma laca, goma arábiga, clara de huevo, colofonia y resina dammar - que se ha sometido a condiciones adversas ambiente de alta humedad, inmersión en agua, acción de temperatura (calor seco) y envejecimiento acelerado, recreados siguiendo normativas internacionales estandarizadas, por considerarlas como representativas de situaciones- como recreación de situaciones representativas de aquellas a las que puede verse expuesta una obra de estas características, ya sea de manera natural, accidental o durante la realización de tratamientos de restauración.

Las posibles variaciones en el color de las probetas han sido estudiadas a partir de los resultados colorimétricos obtenidos en el sistema $C I E L^{*} a^{*} b^{*} 1976$. De la comparación entre las medidas tomadas antes y después de los distintos ensayos se pueden concluir las variaciones en el color y en la luminosidad de los distintos barnices.

\section{Materiales y método}

\section{2.a. Preparación de probetas}

Para la elaboración de las probetas se han seguido los procedimientos de ejecución habituales que se empleaban en este tipo de documentos gráficos barnizados. De acuerdo con esto, se han seleccionado tres materiales: papel, barniz y su correspondiente disolvente.

En primer lugar, se prepararon los barnices siguiendo las recetas tradicionales:

- Goma laca (GL): 1:2 en etanol. Colocar en un bote de cristal y dejar que se disuelva [15].

- Goma arábiga (GA): 1:6 en agua. En un bote de cristal agregar 3 partes de las 6 de goma arábiga que se emplean en la receta final y añadir agua; dejar que se hidrate durante $24 \mathrm{~h}$. Pasado este tiempo, colocar en un agitador magnético para facilitar la mezcla e ir añadiendo poco a poco la goma arábiga restante. Continuar agitando hasta obtener una mezcla homogénea [16].

- Clara de huevo (CH): separar la clara de la yema de un huevo fresco y batir al punto de nieve. Colocar en un bote de cristal y dejarlo reposar durante al menos $12 \mathrm{~h}$. Pasado este tiempo, la clara 
de huevo tendrá una consistencia líquida, lista para usar. Diluir con agua para obtener las distintas concentraciones $[2,15]$.

- Colofonia (CO): 1:2 en etanol. Colocar en un bote de cristal y dejar que se disuelva, agitar cada cierto tiempo para evitar que la formación de grumos [17].

- Dammar (DA): Pulverizar la resina con la ayuda de un mortero y depositarla en un bote de cristal junto con White Spirit en una proporción 1:3. Agitar cada cierto tiempo para facilitar la disolución; filtrar con una gasa antes de su uso [17].

Los barnices así preparados se diluyeron en los disolventes correspondientes hasta obtener barniz a distintas concentraciones (25\%, 50\%, 75\% y 100\%).

Como soporte se ha seleccionado probetas de papel Somerset 100\% algodón, libre de ácidos en un formato de $50 \times 25 \mathrm{~mm}$.

El método de aplicación de los barnices ha sido el aerógrafo. Si bien el número de capas que se aplican en una obra sobre papel no está establecido, pues puede variar en función del acabado deseado y la función de la misma, en nuestro caso dos capas de barniz (Fig.1) fueron suficientes para obtener una superficie barnizada homogéneamente, comprobada mediante la observación de la superficie con microscopio óptico.

Se prepararon cinco lotes de probetas iguales para cada barniz: uno para cada ensayo físico-químico y otro como referencia (probetas de referencia). Cada lote contenía una probeta sin barnizar y una barnizada con cada una de las concentraciones preparadas, lo que permitió comparar las variaciones cromáticas entre las probetas preparadas a distintas concentraciones de barniz y aquellas sin barniz.

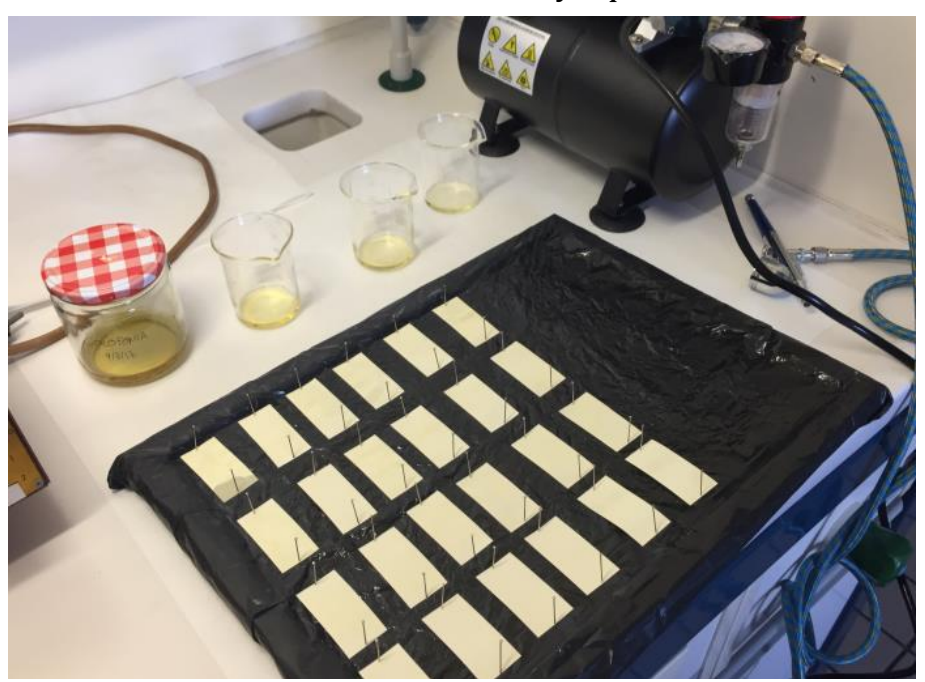

Figura 1. Aplicación mediante aerógrafo del barniz en las probetas.

\section{2.b. Realización de ensayos}

Se han seleccionado cuatro ensayos que se basan en la exposición de las probetas, preparadas según lo indicado en el apartado anterior, a los distintos factores de degradación. Los intervalos de tiempo indicados para cada ensayo siguen las indicaciones de las normas estandarizadas para cada uno de ellos.

\section{Resistencia a la humedad ambiental}

Para conocer los efectos de un ambiente de alta humedad, ya sea a consecuencia del clima o de la aplicación de distintos tratamientos de restauración, las probetas se someten a condiciones de alta humedad hasta la formación de condensación de agua en su superficie, según lo indicado en la Norma UNE-EN ISO 62702:2005 [18]. Para ello, se emplea una cámara de humectación diseñada para su uso en restauración conectada a un humidificador $B U R G B H-840 E$ que utiliza ultrasonidos para generar una fina bruma hasta obtener condensación sobre las probetas. El valor de HR (humedad relativa) mantenido hasta el final del ensayo ha sido del 65\% y los intervalos de estudio de 6, 12, 24 y 48 horas.

\section{Resistencia a la inmersión en agua}

Para conocer los efectos del agua en forma líquida, ya sea como consecuencia de un accidente o desastre natural, o debido a su empleo en tratamientos de restauración, se sumergen las probetas barnizadas en una cubeta con agua, siguiendo las indicaciones de la Norma UNE-EN ISO 2812-2:2007 [19]. Los intervalos de estudio han sido de 30, 60, 90, 120, 150 y 180 minutos. 


\section{Envejecimiento por calor seco}

Para conocer efectos que las altas temperaturas pueden provocar sobre las obras barnizadas - evaporación de compuestos, cambios en las características físicas y ópticas, etc. - se ha utilizado un horno desecador, siguiendo las indicaciones de la Norma UNE-EN ISO 57092-1: 2002, Beschikung-Loading Modell 100-800 Memmert ULE 600 a una temperatura de $105^{\circ}$ [20] a intervalos de 6, 12, 24 y 48 horas.

\section{Envejecimiento en cámara climática}

Para conocer los efectos del envejecimiento acelerado sobre las probetas - cambios en las características físicas y ópticas o variaciones en los niveles de $\mathrm{pH}$ - es necesaria la recreación de unas condiciones adversas de temperatura, humedad y radiación lumínica. Las probetas se colocan en el interior de la cámara climática Solarbox 3000eRH, seleccionando parámetros especificados en la Norma UNE-EN ISO 16474-2:2013 [21]: $80^{\circ} \mathrm{C}$ de temperatura, una humedad relativa del $65 \%$ y una radiación de $550 \mathrm{w} / \mathrm{m}^{2}$. El ensayo se realiza a intervalos de 6, 12, 24 y 48 horas.

\section{2.c. Estudio del color}

El equipo empleado ha sido un espectrofómetro Konica-Minolta CM-2600d, empleando el espacio de color CIE $L^{*} a^{*} b^{*} 1976$, con las siguientes condiciones de medición: geometría de medición de/8 (iluminación difusa, ángulo de visión de $8^{\circ}$ ), reflexión especular excluida, intervalo de longitudes de onda de $360 \mathrm{~nm}$ a $740 \mathrm{~nm}$, paso de longitud de onda de $10 \mathrm{~nm}$, lámparas pulsatorias de xenón como fuentes de luz, tiempo de medida de 1,5 segundos aproximadamente, diámetro de apertura de $8 \mathrm{~mm}$, iluminación $0 \%$ de UV, observador patrón CIE 1964 de $10^{\circ}$ e iluminante patrón CIE D65. Para el valor de cromaticidad, la desviación estándar $\Delta E_{a b}{ }^{*}$ está dentro del $0,04 \%$. Para el estudio de los datos obtenidos se ha empleado el software de control del color Color Data Software CM-S100W Spectramagic ${ }^{\mathrm{TM}}$ NX Pro versión 2.4.

Se tomaron 3 medidas en distintas áreas de la probeta, de las que se sacará el promedio de los valores $L^{*}$ (luminosidad), $a^{*}$ (eje rojo-verde) y $b^{*}$ (eje azul-amarillo), a partir de los cuales se calculará el valor de diferencia total $\Delta E_{a b}{ }^{*}$ a partir de la siguiente ecuación:

$$
\Delta E_{a b} *=\left(\left(\Delta L^{*}\right)^{2}+\left(\Delta a^{*}\right)^{2}+\left(\Delta b^{*}\right)^{2}\right)^{1 / 2}
$$

Donde: $\Delta L^{*}$ es la diferencia entre el valor $L^{*}$ de referencia y el de la probeta de ensayo; $\Delta a^{*}$ es la diferencia entre el valor $a^{*}$ de referencia y el de la probeta de ensayo; y $\Delta b^{*}$ es la diferencia entre el valor $b^{*}$ de referencia y el de la probeta de ensayo [22].

Para este estudio se tendrá en cuenta que habrá diferencias perceptibles de color cuando los valores de diferencia total $\left(\Delta E_{a b}{ }^{*}\right)$ sean iguales o superiores a 2 [23], en tanto que las variaciones por debajo de este valor se consideran inapreciables. Aunque este umbral de tolerancia no ha sido determinado específicamente para papeles barnizados, la falta de estudios al respecto ha supuesto su toma en consideración como valor indicativo de un caso general.

Para el estudio de los datos obtenidos se ha descartado la comparativa de datos promediados a favor de la relación entre los datos de las probetas de referencia y los datos de las probetas tras cada uno de los ensayos realizados.

\section{Resultados y discusión}

Siguiendo la metodología indicada anteriormente, se ha estudiado el color de los distintos lotes de probetas para cada barniz.

De manera general, la observación de los barnices aplicados sobre las probetas tras la realización de los ensayos, demuestra que la variación de color más habitual es la tendencia a tonalidades más amarillas. Ese "amarilleamiento" se observa al aumentar significativamente el valor $b^{*}$ mientras las otras coordenadas, $\mathrm{L}^{*}$ y a*, no varían significativamente; si, por el contrario, $L^{*}$ baja o $a^{*}$ sube más de lo que cambia $b^{*}$, el color del barniz tiende al rojo oscuro.

\section{Probetas de referencia}

El estudio colorimétrico de las probetas de referencia sin barnizar (0\%) muestra que no hay cambios a considerar que puedan interferir en los valores de las capas de barniz. Presentan unos valores de $\mathrm{L}^{*}$ y a* similares, mientras que b* es prácticamente idéntico en todos los casos (Tabla 1). 
Tabla 1. Valores CIELab L*a* y b* para las probetas de referencia sin barnizar para cada uno de los lotes, así como el promedio (AVG) de las distintas medidas.

\begin{tabular}{|l|c|c|c|}
\hline & $\boldsymbol{L}^{*}$ & $\boldsymbol{a}^{*}$ & $\boldsymbol{b}^{*}$ \\
\hline \hline GL 0\% & 96,14 & $-2,03$ & 8,49 \\
\hline GA 0\% & 96,25 & $-2,12$ & 8,38 \\
\hline CH 0\% & 95,83 & $-1,82$ & 8,61 \\
\hline CO 0\% & 95,95 & $-1,58$ & 8,16 \\
\hline DA 0\% & 96,11 & $-1,85$ & 8,25 \\
\hline \hline AVG & 96,06 & $-1,88$ & 8,38 \\
\hline
\end{tabular}

El color de todos los barnices se acentúa al aumentar la concentración de barniz. La intensificación del color queda reflejada en el aumento de los valores $\mathrm{a}^{*}$ y b*; además, su color tiende a oscurecerse, es decir, a disminuir el valor $L^{*}$ de manera general, salvo en el caso del barniz de clara de huevo (Tabla 2).

Tabla 2. Valores CIELab $L^{*} a^{*}$ y $b^{*}$ para las probetas de referencia con barniz de goma laca (GL), goma arábiga (GA), clara de huevo $(\mathrm{CH})$, colofonia (CO) y dammar (DA) a distintas concentraciones.

\begin{tabular}{|c|c|c|c|c|}
\hline Barniz & Concentración (\%) & $L^{\prime *}$ & $\overline{a^{*}}$ & $\boldsymbol{b}^{*}$ \\
\hline \multirow{5}{*}{ Goma laca } & 0 & 96,14 & $-2,03$ & 8,49 \\
\hline & 25 & 95,17 & $-3,22$ & 14,64 \\
\hline & 50 & 94,91 & $-3,51$ & 15,97 \\
\hline & 75 & $\begin{array}{l}94,38 \\
\end{array}$ & $-3,75$ & 18,12 \\
\hline & 100 & 94,22 & $-3,69$ & 18,12 \\
\hline \multirow{5}{*}{ Goma arábiga } & 0 & 96,25 & $-2,12$ & 8,38 \\
\hline & 25 & 95,48 & $-2,55$ & 10,84 \\
\hline & 50 & 95,48 & $-2,27$ & 10,13 \\
\hline & 75 & 95,49 & $-2,51$ & 10,29 \\
\hline & 100 & 95,50 & $-2,30$ & 10,21 \\
\hline \multirow{5}{*}{ Clara de huevo } & 0 & 95,83 & $\begin{array}{ll}-1,82 \\
\end{array}$ & 8,61 \\
\hline & 25 & 95,19 & $-2,25$ & 11,22 \\
\hline & 50 & 95,34 & $-2,49$ & 12,02 \\
\hline & 75 & 95,32 & $-2,44$ & 11,25 \\
\hline & 100 & 95,27 & $-2,37$ & 11,18 \\
\hline \multirow{5}{*}{ Colofonia } & 0 & 95,95 & $-1,58$ & 8,16 \\
\hline & 25 & 94,74 & $-2,86$ & 13,30 \\
\hline & 50 & 94,32 & $-3,13$ & 16,50 \\
\hline & 75 & 94,18 & $-3,31$ & 16,91 \\
\hline & 100 & 93,92 & $-3,53$ & 18,61 \\
\hline \multirow{5}{*}{ Dammar } & 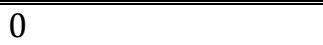 & 96,11 & $-1,85$ & 8,25 \\
\hline & 25 & 95,34 & $-2,42$ & 11,98 \\
\hline & 50 & 95,04 & $-2,83$ & 13,37 \\
\hline & 75 & 94,60 & $-2,97$ & 14,57 \\
\hline & 100 & 94,30 & $-3,15$ & 15,71 \\
\hline SD & & 0,02 & 0,01 & 0,04 \\
\hline
\end{tabular}

Los barnices presentan unos valores $\mathrm{L}^{*}$ y a* similares entre sí. La luminosidad de los barnices es alta, con valores de $\mathrm{L}^{*}$ superiores a 90 en todos los casos; en el caso de $\mathrm{a}^{*}$, todos los barnices presentan valores que se sitúan entre -1 y -4 , mostrando así una tendencia hacia tonalidades verdes, pero siempre en valores neutros (cercanos al acromático). Las mayores diferencias entre los barnices se dan en los valores de $b^{*}$ :

- Los barnices de goma laca y colofonia son más amarillos que los demás, especialmente en concentraciones altas ya que su valor $b^{*}$ es más elevado, con un máximo de 18,12 para la goma laca y 18,61 para la colofonia.

- El barniz dammar presenta una tonalidad menos amarilla que los anteriores, con un valor máximo de 15,71 . 
- Los barnices de goma arábiga y clara de huevo son menos amarillos, con una tonalidad más neutra y cercana a la del papel, mostrando unos valores $b^{*}$ máximos de 10,84 para la goma arábiga y de 12,02 para la clara de huevo (Fig. 2 y Tabla 2 ).

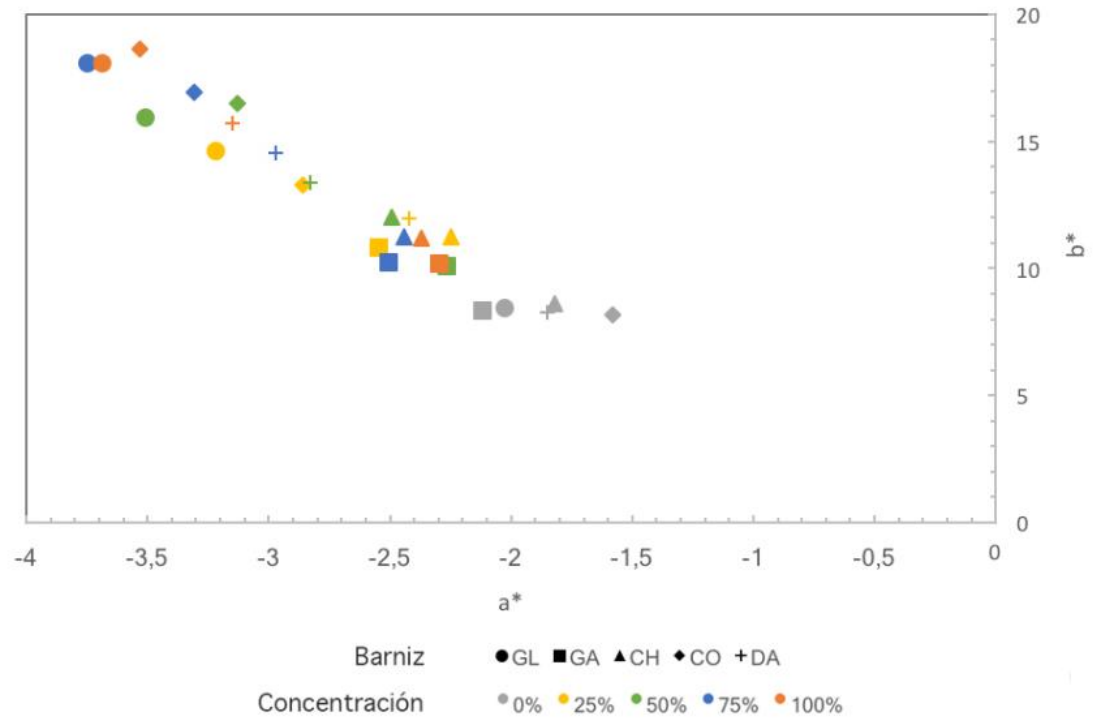

Figura 2. Coordenadas cromáticas $a^{*}$ y $b^{*}$ de las muestras de referencia de goma laca (GL), goma arábiga (GA), clara de huevo (CH), colofonia (CO) y dammar (DA) a distintas concentraciones.

\section{Resistencia a la humedad ambiental}

El estudio del color de las probetas tras este ensayo no muestra variaciones en los barnices de goma laca, goma arábiga y dammar, así como en las probetas sin barnizar, ya que en todos los casos encontramos unas diferencias totales $\left(\Delta \mathrm{Eab}^{*}\right.$ ) inferiores a 2 , por lo que la humedad no ha alterado su color. En el caso de los barnices a base de clara de huevo y colofonia, las variaciones se traducen en un aumento de $b^{*}$ con respecto a los valores de referencia (Fig. 3):

- En el caso de la clara de huevo, estas diferencias en el color sólo se dan en las probetas con una concentración de barniz del 100\% a partir de las 24 horas de ensayo. Esta variación se traduce en un aumento progresivo de $b^{*}$ desde un valor inicial de 11,18 hasta 13,85 tras $48 \mathrm{~h}$ de ensayo. Esto indica un ligero amarilleamiento del barniz de clara de huevo al 100\% de concentración después de estar sometido $48 \mathrm{~h}$ a humedad ambiental.

- Las probetas con colofonia presentan cambios perceptibles de color a concentraciones del 25\%, $50 \%$ y $75 \%$, apreciándose en este caso oscilaciones en las medidas. Los datos obtenidos para el valor $b^{*}$ muestran que a un 25\% de concentración aumenta a las 6 h, pasando de 13,30 a 16,99, valor que se mantiene similar hasta finalizar el ensayo. Las probetas con colofonia al $50 \%$ no muestran cambios en el color hasta las $48 \mathrm{~h}$ de ensayo, con un aumento hasta 19,42; por último, las probetas con un $75 \%$ únicamente presentan cambios en $b^{*}$ al inicio del ensayo $(6 \mathrm{~h})$, pasando de 16,91 hasta 20,41 .

Esto indica que los barnices de clara de huevo y colofonia muestran una tendencia general hacia tonalidades más amarillas. 
(a)
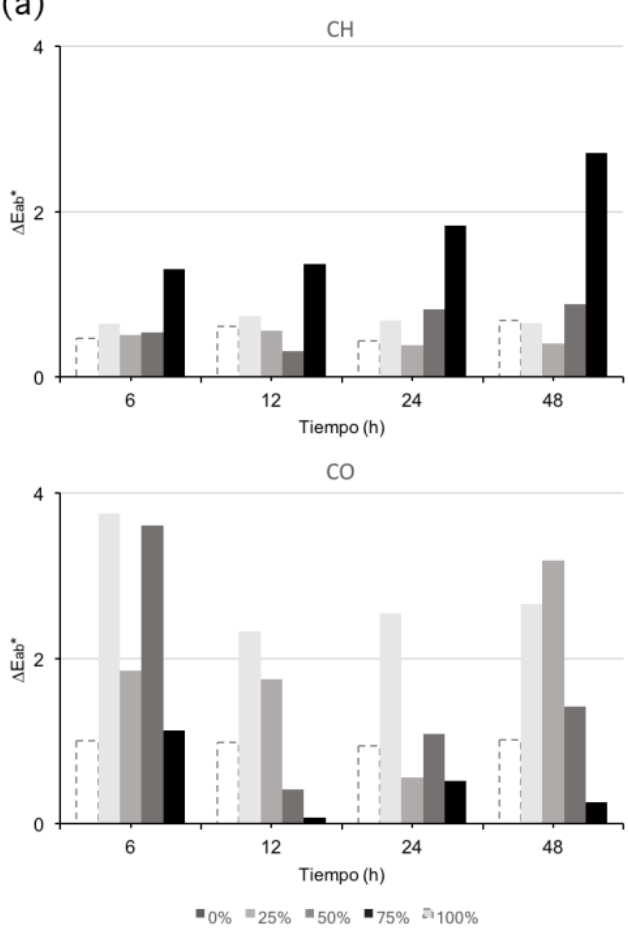

(b)

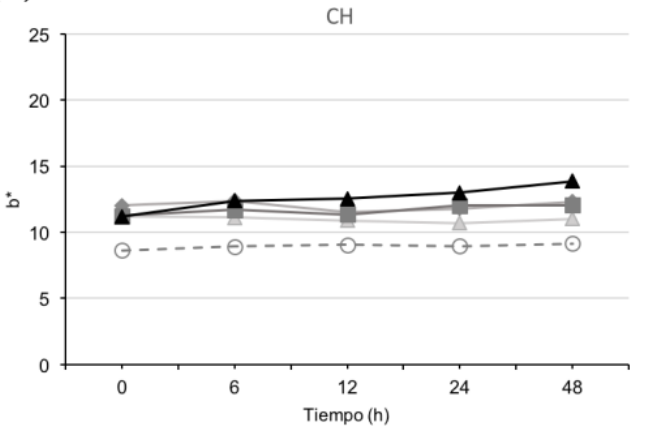

$\mathrm{CO}$

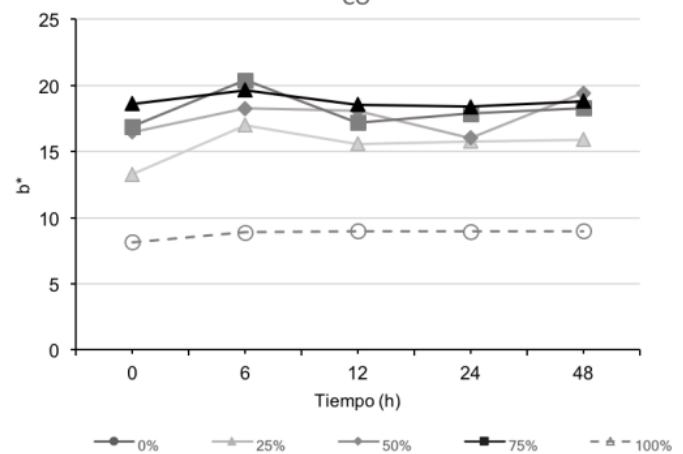

Figura 3. Resultado del estudio colorimétrico para las probetas con barniz de clara de huevo (CH) y colofonia (CO) a distintas concentraciones, sometidas al ensayo de resistencia a la humedad ambiental. (a) diferencias colorimétricas totales ( $\left.\Delta E_{a}{ }^{*}\right)$; y (b), valores $b^{*}$

\section{Resistencia a la inmersión en agua}

Este ensayo afecta claramente el comportamiento cromático de los barnices pues, en todos ellos se han dado diferencias perceptibles de color para algunas concentraciones e intervalos de tiempo, con diferencias totales $\left(\Delta E_{a b}{ }^{*}\right)$ superiores a 2 (Fig. 4 ):

- En el caso de la goma laca estas diferencias se dan al final del ensayo para concentraciones del $50 \%$ y $75 \%$, que se traducen en una disminución de $b^{*}$ hasta unos valores de 13,83 y 16,15 , respectivamente; en el caso de las probetas con un 100\%, la mayor disminución se da también al final del ensayo, hasta 14,91 . Los valores $L^{*} \mathrm{y} a^{*}$ permanecen constantes durante el ensayo en todas las concentraciones.

- Para la goma arábiga solo se dan diferencias perceptibles superiores a 2 a una concentración del $25 \%$ a partir de $90 \mathrm{~min}$. El valor $b^{*}$ de las probetas barnizadas muestra una tendencia a igualarse entre las probetas barnizadas hasta valores similares a los de la probeta sin barnizar (entre 8,57 y 9,19).

- Las probetas con clara de huevo presentan variaciones en todas las probetas barnizadas, que se inician a los $30 \mathrm{~min}$ y se hacen constantes hasta el final del ensayo. Al igual que en el caso de la goma laca, los valores $b^{*}$ tienden a igualarse entre las probetas barnizadas hasta valores similares a los de la probeta sin barnizar $(8,96$ y 9,50). En ambos casos, esto muestra la solubilidad de estos materiales en el agua, llegando a ser eliminados al inicio del ensayo. Estos resultados se han corroborado con la realización paralela de otros estudios, como son la medida del pH, del brillo y la observación de la superficie con microscopio óptico.

- Al igual que ocurre en el ensayo de resistencia a la humedad ambiental, los resultados para el barniz de colofonia presentan oscilaciones, con variaciones en las probetas con una concentración del $50 \%$ y $100 \%$ de barniz. Se observan una disminución de $b^{*}$ en estas concentraciones: a un $50 \%$ de colofonia los valores oscilan entre 13,96 y 16,44 ; en el caso de las probetas con un $100 \%$, este valor aumenta de manera progresiva hasta un valor final de 15,99.

- Solo se observan cambios en el barniz dammar en las probetas con un 100\% a partir de 150 min; en este caso, el valor $b^{*}$ tiende a disminuir, pasando de un valor de 15,71 de la muestra de referencia hasta 13,59 a los 180 min de ensayo (Fig.4). 
(a)

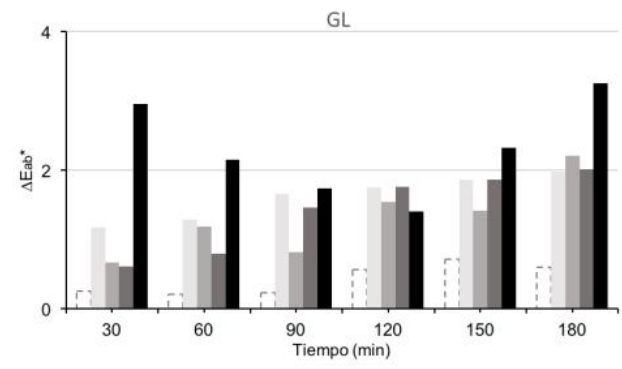

GA

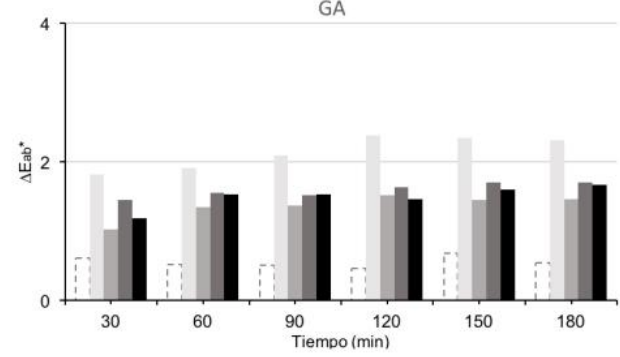

$\mathrm{CH}$

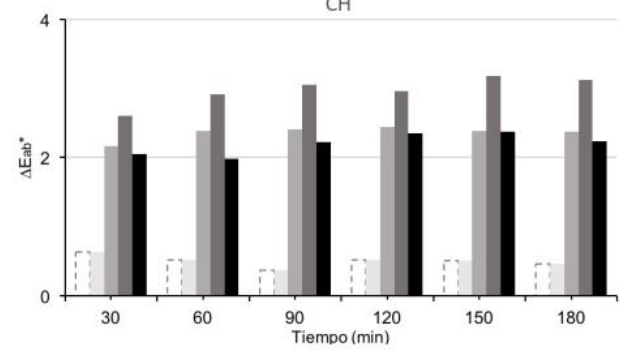

$\mathrm{CO}$

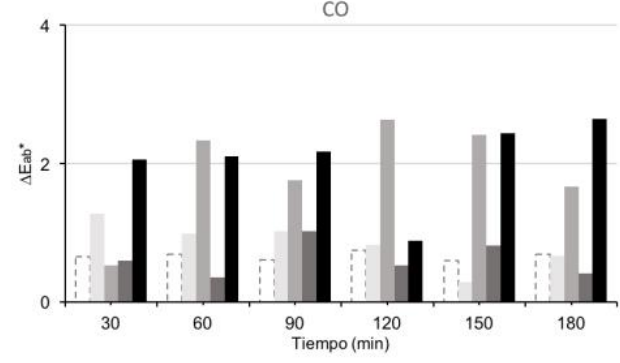

DA

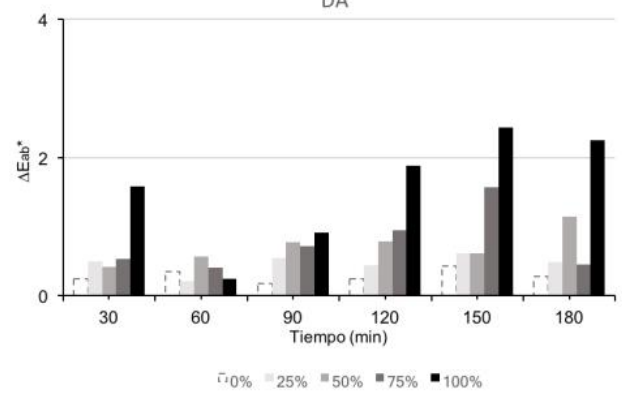

(b)

GL

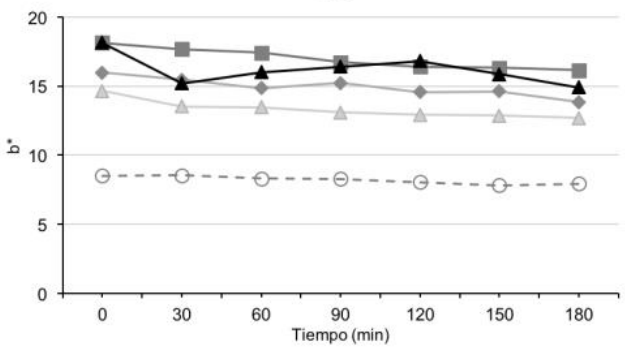

GA
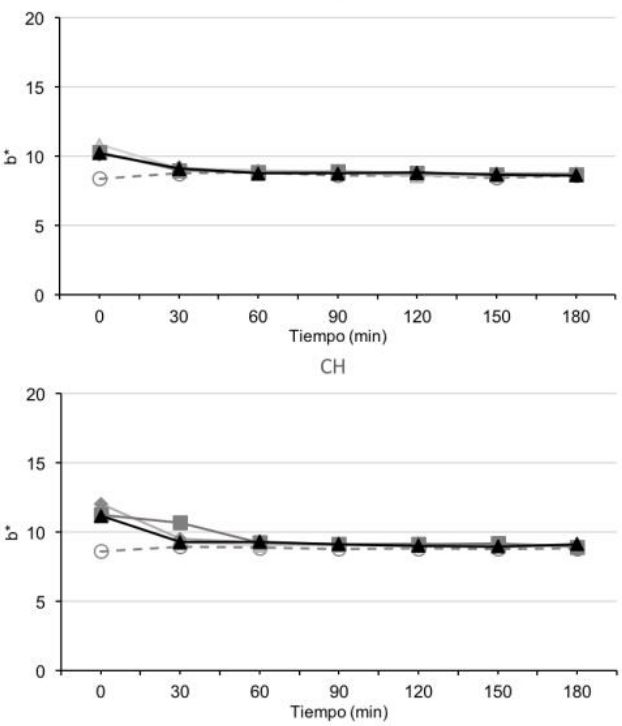

CO
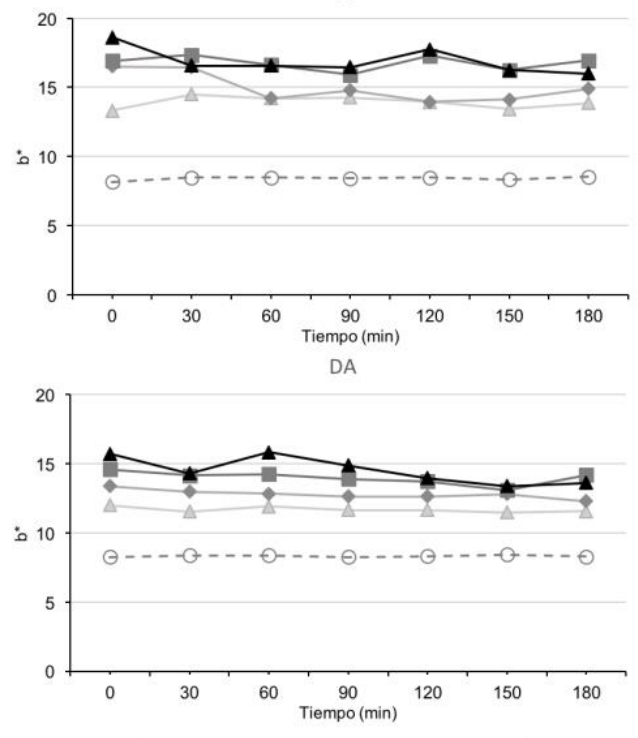

$-\theta-0 \% \rightarrow 25 \% \rightarrow-50 \% \rightarrow-75 \% \rightarrow-100 \%$

Figura 4. Resultado del estudio colorimétrico para las probetas con barniz de goma laca (GL), goma arábiga (GA), clara de huevo $(\mathrm{CH})$, colofonia (CO) y dammar (DA) a distintas concentraciones, sometidas al ensayo de resistencia a la inmersión en agua. (a) diferencias colorimétricas totales $\left(\Delta \mathrm{Eab}^{*}\right) ; \mathrm{y}(\mathrm{b})$, valores $\mathrm{b}^{*}$.

\section{Envejecimiento por calor seco}

La exposición de las probetas a una temperatura elevada durante un tiempo máximo de $48 \mathrm{~h}$ ha tenido efecto en el color de todos los barnices estudiados con la excepción de la goma arábiga y de las probetas sin barnizar, ya que encontramos diferencias colorimétricas totales $\left(\Delta \mathrm{Eab}^{*}\right)$ superiores a 2 en la mayor parte 
de casos. De nuevo, estas variaciones han tenido lugar en el valor $\mathrm{b}^{*}$, con una tendencia general a aumentar en todas las probetas barnizadas con goma laca, clara de huevo, colofonia y dammar:

- La goma laca presenta diferencias perceptibles con respecto a las muestras de referencia únicamente a concentraciones del $50 \%$ y $75 \%$, a partir de $12 \mathrm{~h}$ y $6 \mathrm{~h}$ respectivamente. Las probetas con un $75 \%$ son las que presentan unos valores $b^{*}$ más elevados, superior al de las probetas con barniz a su máxima concentración, hasta un valor máximo de 22,64 a las 48 h de ensayo frente a un 17,33 de la probeta con un $100 \%$ en el mismo intervalo de tiempo.

- La clara de huevo muestra diferencias perceptibles en las probetas con una concentración superior al 50\%, que aumentan progresivamente a lo largo del ensayo, siendo mayores para las probetas con un $100 \%$ de clara de huevo. Se aprecian oscilaciones en el valor $b^{*}$ en las probetas con un $50 \%$ y un $75 \%$ barniz; los mayores cambios se dan a un $100 \%$, también con un aumento progresivo de $b^{*}$ hasta un valor final de 18,66 .

- Se observan diferencias en todas las concentraciones de barniz de colofonia. Este barniz muestra los valores $b^{*}$ más elevados, que aumentan progresivamente en todas las probetas barnizadas hasta unos valores máximos de 19,06 (25\%), 23,93 (50\%), 25,91 (75\%) y 27,66 (100\%); como se puede apreciar, el valor $b^{*}$ es, además, mayor conforme aumenta la concentración de colofonia.

- Al igual que en el caso de la colofonia, el barniz dammar muestra diferencias perceptibles $\left(\Delta E_{a b}{ }^{*}\right)$ superiores a 2 en todas las concentraciones. Sin embargo, al contrario que en el caso anterior, para el barniz dammar, se aprecia una disminución inicial de $b^{*}$ en las probetas con una concentración del 50\%, aunque finaliza el ensayo con valores similares a los de referencia $(18,02)$; a concentraciones del $25 \%$ y $75 \%$ la tendencia es a aumentar, finalizando el ensayo con unos valores de 17,97 y 21,45 respectivamente; finalmente, en el caso de las probetas con barniz a su máxima concentración, no se observan variaciones durante las primeras $12 \mathrm{~h}$ de ensayo, momento en el que empieza a disminuir hasta un valor final de 11,65 (Fig. 5).

\section{Envejecimiento en cámara climática}

$\mathrm{Al}$ igual que en el ensayo anterior, la goma arábiga no se ha visto afectada al no presentar cambios en su color, salvo mínimas variaciones a una concentración del 25\% En el resto de barnices se aprecian diferencias totales $\left(\Delta \mathrm{Eab}^{*}\right)$ mayores de 2 en todas las probetas barnizadas, (aunque no en todas las concentraciones) no observándose cambios en las probetas sin barnizar. Al igual que en el caso de los ensayos anteriores, estos cambios en el color tienen lugar en el valor $\mathrm{b}^{*}$, con una tendencia general a aumentar, es decir, a amarillear, en los barnices de goma laca y colofonia:

- El barniz de goma laca presenta oscilaciones a concentraciones del 25\%, 50\% y 75\%, mientras que a un $100 \%$ estas variaciones aumentan a partir de las $12 \mathrm{~h}$. Si bien se aprecian variaciones en las probetas con concentraciones del $25 \%, 50 \%$ y $75 \%$, los valores $b^{*}$ al final del ensayo son similares a los de las muestras de referencia, mientras que a un $100 \%$ se aprecia un aumento progresivo de $b^{*}$ hasta un valor máximo de 27,53 .

- El barniz de clara de huevo muestra diferencias con respecto al valor inicial al final del ensayo para concentraciones del $25 \%, 75 \%$ y $100 \%$, mientras que para un 50\% estas variaciones son mayores y aumentan de manera progresiva desde el inicio del ensayo. El barniz de clara de huevo es el único en el que el valor $b^{*}$ muestra una tendencia a disminuir, finalizando el ensayo con valores similares para todas las concentraciones -entre 7,25 y 8,86 .

- Las mayores diferencias se dan en el barniz de colofonia, con variaciones en todas las probetas que tienden a acentuarse conforme aumenta el tiempo de exposición en la cámara climática, siendo superiores para concentraciones del $75 \%$ y del $100 \%$. El valor $b^{*}$ muestra un aumento progresivo a lo largo del ensayo, salvo en el caso de la probeta con un $75 \%$ que presenta una disminución de $b^{*}$ a las $6 \mathrm{~h}$ y un aumento progresivo a partir de las $12 \mathrm{~h}$; los valores finales para este barniz son $20,88(25 \%), 23,59(50 \%), 27,54(75 \%)$ y $27,68(100 \%)$, es decir, que este valor tiende a aumentar no solo durante el ensayo sino también conforme aumenta la concentración de barniz, encontrando además valores similares para las probetas con concentraciones del $75 \%$ y del $100 \%$.

- En el caso del dammar, únicamente se aprecian diferencias perceptibles en las probetas con una concentración del $100 \%$ a partir de las 12 h de ensayo. Estas probetas muestran una disminución de $b^{*}$ progresiva hasta las $12 \mathrm{~h}$, pasando de un valor inicial de 15,71 a 10,69, momento en que se estabiliza hasta las $48 \mathrm{~h}$; de este modo, las probetas con barniz a su máxima concentración presentan un valor $b^{*}$ inferior al de las probetas con concentraciones del 25\%, 50\% y 75\% (Fig. 6.b). 
(a)

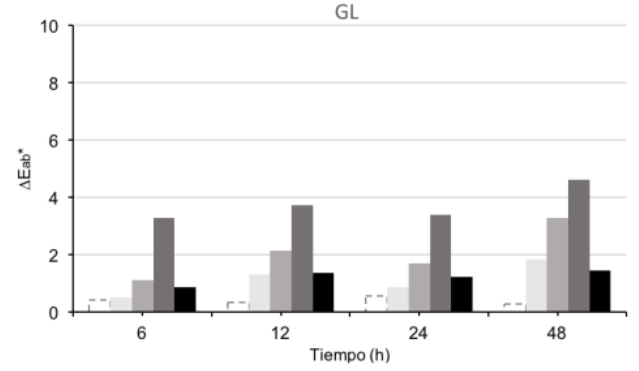

GA

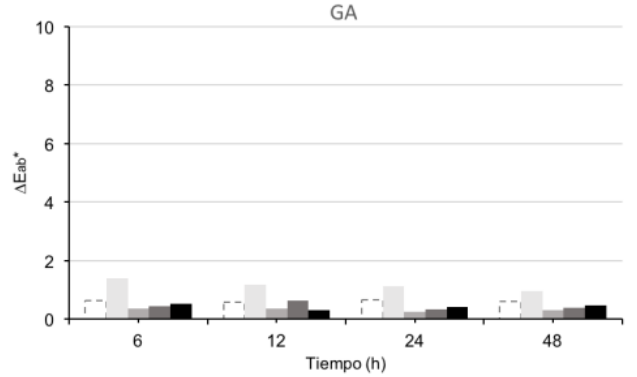

$\mathrm{CH}$

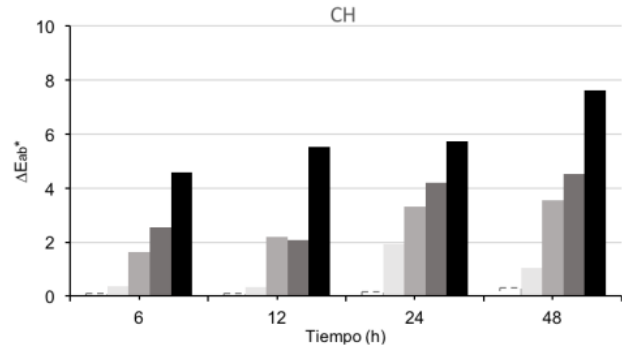

CO

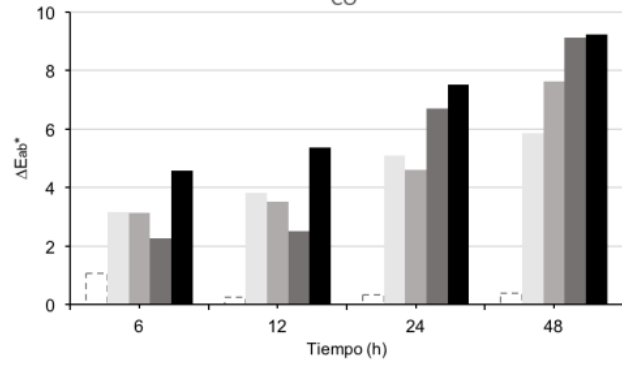

DA

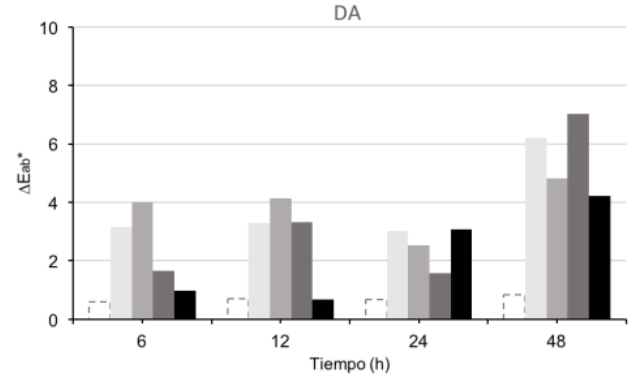

Fo\% $=25 \%=50 \%=75 \%-100 \%$ (b)
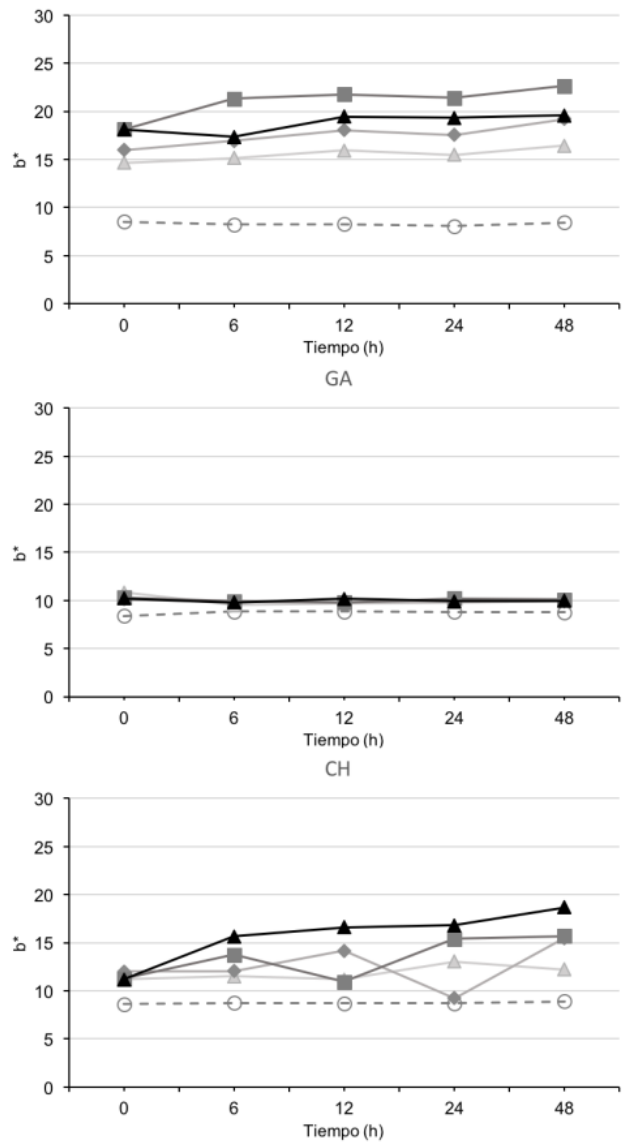

$\mathrm{CO}$
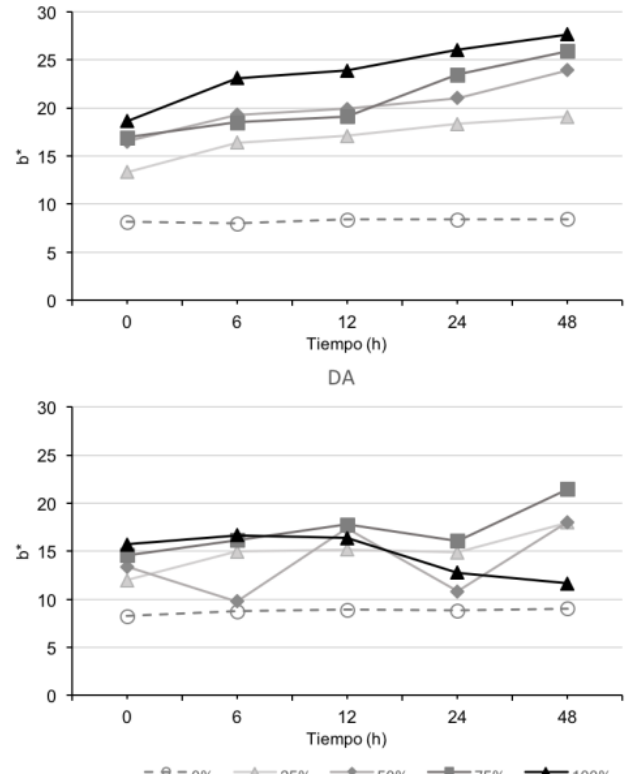

Figura 5. Resultado del estudio colorimétrico para las probetas con barniz de goma laca (GL), clara de huevo (CH), colofonia (CO) y dammar (DA) a distintas concentraciones, sometidas al ensayo de envejecimiento por calor seco. (a) diferencias colorimétricas totales $\left(\Delta E_{a b}{ }^{*}\right) ; \mathrm{y}(\mathrm{b})$, valores $b^{*}$. 
(a)

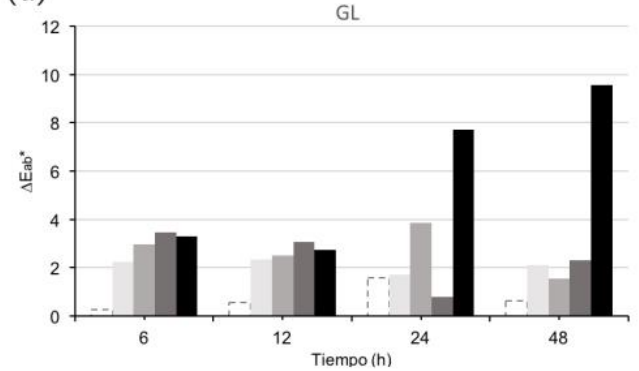

GA

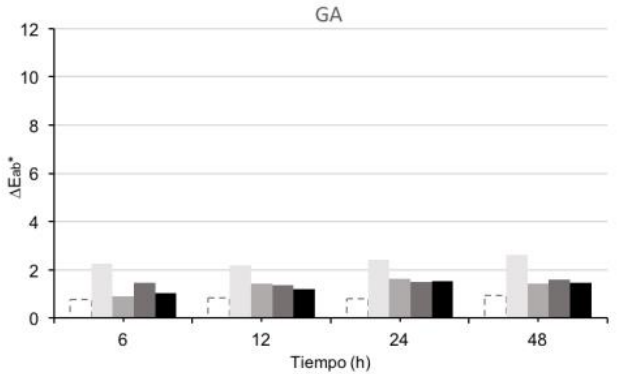

$\mathrm{CH}$

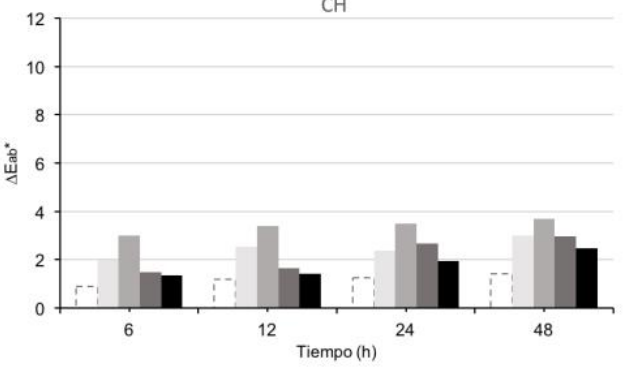

CO

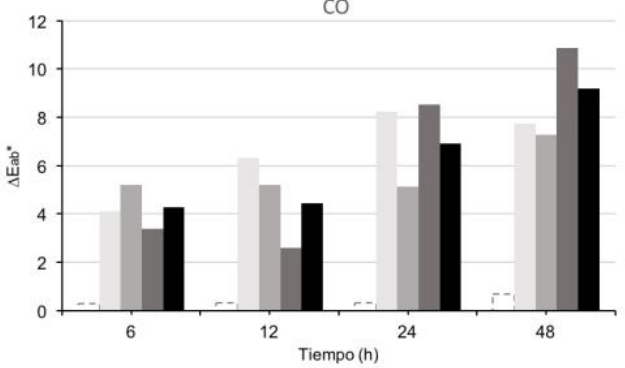

DA

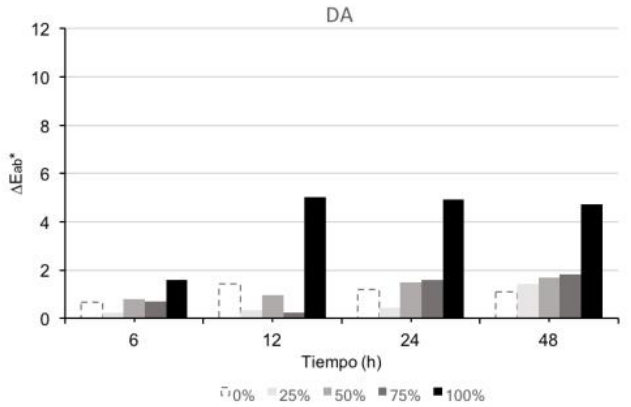

(b) GL

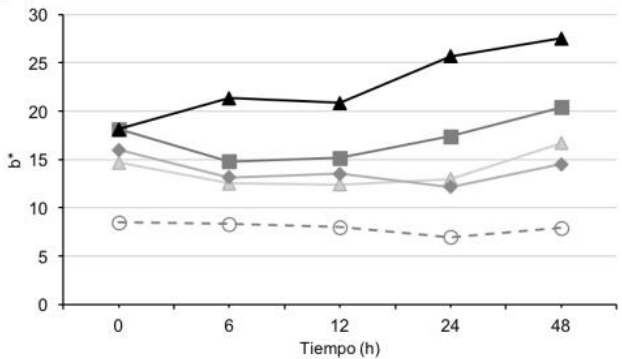

GA
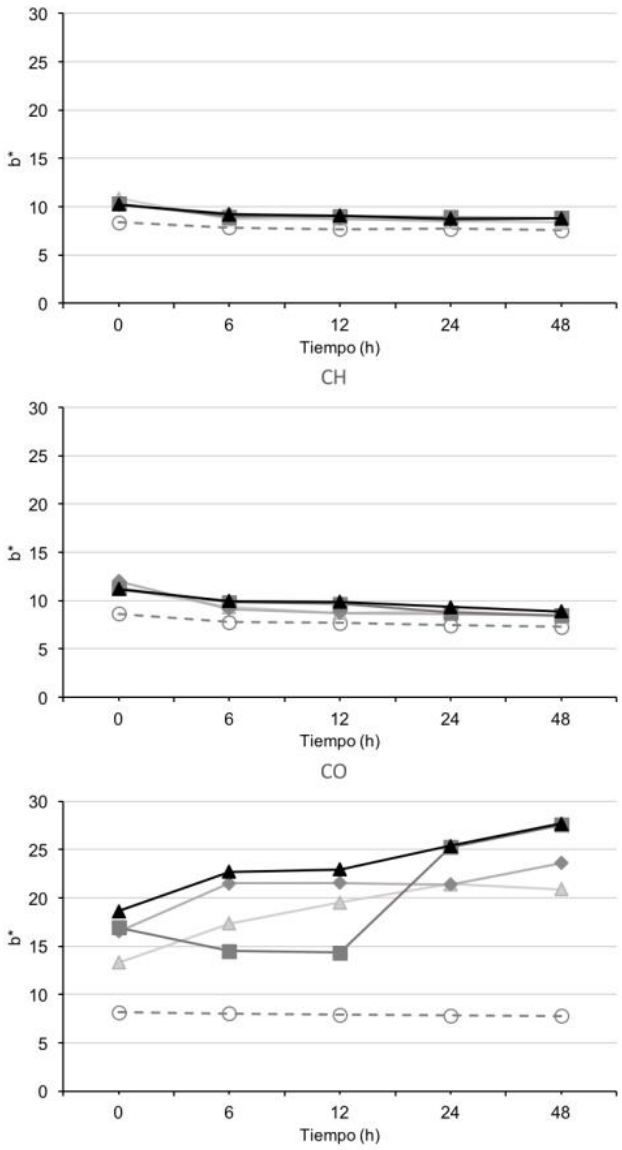

DA

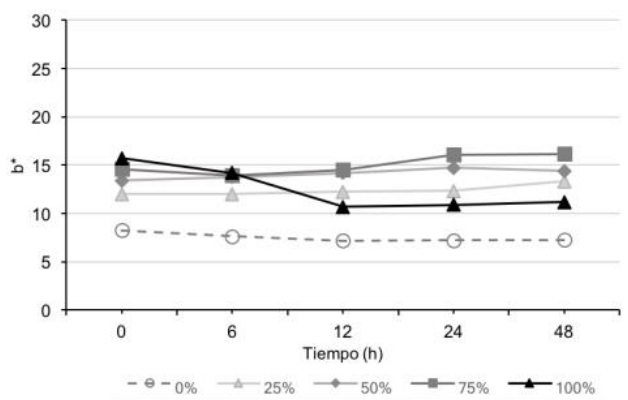

Figura 6. Resultado del estudio colorimétrico para las probetas con barniz de goma laca (GL), goma arábiga (GA), clara de huevo $(\mathrm{CH})$, colofonia (CO) y dammar (DA) a distintas concentraciones, sometidas al ensayo de envejecimiento en cámara climática. (a) diferencias colorimétricas totales $\left(\Delta E_{a b}{ }^{*}\right)$; y (b), valores $b^{*}$. 


\section{Conclusiones}

De manera general, las variaciones en el color de las probetas se acentúan conforme aumenta la concentración del barniz

Estas variaciones se han producido en todos los casos en el valor $b^{*}$, manteniéndose los valores de $L^{*}$ y $a^{*}$ estacionarios. En el caso del valor $L^{*}$, la tendencia de evolución es mínima y se traduce en una pérdida de luminosidad, aunque manteniendo siempre niveles superiores a 90; el valor $a^{*}$ también disminuye, aunque muy ligeramente y dentro de valores neutros. Esto se indica que los cambios en el color de los barnices se han producido por un aumento o disminución de la tonalidad amarilla.

El estudio del color de los barnices recién aplicados (probetas de referencia) indican que los barnices de goma laca, colofonia y dammar tienen una tonalidad amarilla desde el inicio, por lo que ya desde su aplicación modifican el color subyacente, mientras que los barnices a base de agua -goma arábiga y clara de huevo- son incoloros por lo que no interfieren en el color de los estratos inferiores.

Por ensayos, la humedad ambiental y la inmersión en agua son los que han afectado en menor medida al color de las probetas, con ligeras disminuciones del valor $b^{*}$ en los barnices de goma arábiga, clara de huevo y dammar. En el caso de la inmersión en agua, los valores $b^{*}$ de la goma arábiga y la clara de huevo tras el ensayo son similares a los de las muestras de referencia, lo que indicaría su desaparición durante el ensayo lo que hace lógico los resultados observados ya que ambos son solubles en agua.

Los cambios más significativos, es decir, las mayores diferencias totales con respecto a las probetas de referencia, se han producido tras el envejecimiento acelerado de las probetas. El envejecimiento por calor seco ha afectado en mayor medida a la clara de huevo, la colofonia y la resina dammar, mientras que el envejecimiento en cámara climática ha afectado especialmente a los barnices de goma laca y colofonia. En la mayoría de los barnices, estas variaciones se han producido en el valor de $b^{*}$, con un aumento general de este valor, con la excepción de la resina dammar tras su envejecimiento en cámara climática, en la que se observa una disminución de este valor. A la vista de estos resultados, podemos indicar que la temperatura y la radiación UV son los factores que más alteran el color de los barnices, produciendo su amarilleamiento.

Desde el punto de vista colorimétrico, los resultados obtenidos tras este estudio muestran que la goma arábiga es muy estable a diferentes factores de degradación, especialmente a aquellos implicados en el envejecimiento acelerado (temperatura, humedad e iluminación combinados), aunque se ha comprobado su disolución total durante el ensayo de resistencia a la inmersión en agua; de modo que este barniz es estable a la temperatura y la radiación, pero sensible al agua líquida. Por el contrario, el barniz de colofonia es el que presenta más variaciones en su color para todos los ensayos, con una importante tendencia a amarillear, es decir a aumentar el valor $b^{*}$, tras su envejecimiento; por su parte, la goma laca también muestra una tendencia a aumentar su tonalidad amarilla, pero en menor medida que la colofonia y solo a concentraciones altas de barniz.

En resumen, tanto el barniz de colofonia como el de goma laca han demostrado que su color varía cuando son expuestos a temperatura elevada y a radiación UV. El barniz de clara de huevo se altera en presencia de agua -humedad ambiental e inmersión en agua- y tras la exposición a temperatura elevada, mientras que el color de la resina dammar se ve afectada tras la inmersión en agua, el envejecimiento por calor seco y el envejecimiento en cámara climática, es decir, al agua, la temperatura y la radiación UV.

Los resultados obtenidos durante este estudio pueden ayudar a la selección de los barnices más estables desde el punto de vista colorimétrico con vistas a su restitución como acabado final en los tratamientos de conservación y restauración de documentos gráficos. En este sentido, el barniz de goma arábiga es el que presenta mejores resultados ante la humedad ambiental y el envejecimiento acelerado; tanto por calor seco como en cámara climática, mientras que el dammar es el que mejor resiste a la inmersión en agua.

Esta investigación se podría ampliar en el futuro con otros barnices empleados como materiales contemporáneos para restauración o protección de obra gráfica, con el estudio de otros factores que pueden tener un efecto directo en el color de las probetas, como el número de capas de barniz aplicadas o la influencia del soporte. Además, el análisis podría ampliarse mediante el empleo de imágenes espectrales que permitan obtener mayor información en relación con la textura, variabilidad espacial dentro de un misma muestra o su estado de conservación y la identificación de las alteraciones que puedan presentar. 


\section{Agradecimientos}

Este trabajo fue realizado dentro del proyecto (MAT2014-58659-P) "Nuevas alternativas al conocimiento de los materiales y los procesos de conservación y restauración de obra gráfica y patrimonio documental" financiado por el Ministerio de Economía y Competitividad y por el Fondo Europeo de desarrollo regional FEDER.

Así mismo, agradecer al doctor Francisco Collado Montero (Dpto. Pintura, Universidad de Granada) su colaboración en la toma y registro de datos. 